\title{
The Phonon Density of States Measured with Synchrotron Radiation and Nuclear Resonances
}

Wolfgang Sturhahn, Michael Hu, Sarvjit Shastri, and Tom Toellner

Advanced Photon Source, Argonne National Laboratory

9700 South Cass Ave., Argonne, IL 60439 



\section{Contents}

$\begin{array}{ll}\text { Overview } & 1\end{array}$

$\begin{array}{ll}\text { Principles of the Method } & 1\end{array}$

Setup and Components $\quad 4$

X-ray source . . . . . . . . . . . . . . . . . . . 5

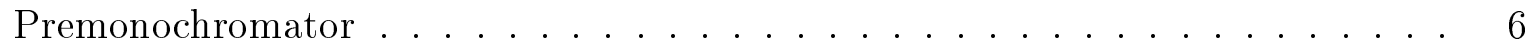

High-resolution monochromator . . . . . . . . . . . . . . . . 6

Detector and timing circuit . . . . . . . . . . . . . . . 9

Experimental Procedure $\quad 11$

Checklist for collecting data . . . . . . . . . . . . . . . 11

$\begin{array}{ll}\text { Additional Reading } & 12\end{array}$

$\begin{array}{ll}\text { Quiz } & 13\end{array}$ 


\section{Overview}

In this experiment, we will use synchrotron radiation to measure the density of states of vibrational excitations (phonons.) Each group of students will conduct an experiment at sector 3-ID of the Advanced Photon Source, the nation's premier synchrotron radiation facility. We provide one support staff per group, i.e., Drs. Michael Hu, Sarvjit Shastri, Wolfgang Sturhahn, and Tom Toellner will help their group to perform the experiment and interpret the data. After data collection (1-2 h per group), the remaining time will be spent with evaluation and interpretation. In addition to your own data, we provide similar sets of data. Computer hardware (iMac running as X-terminals) and software for data manipulation will be provided. It is important that you understand the basic principles of the experimental method. Therefore we strongly recommend that you read the next section and the attached article Phonon Density of States Measured by Inelastic Nuclear Resonant Scattering. You are expected to use this description to familiarize yourself with the experimental setup and its individual components before the start of the experiment. You should be able to solve at least $75 \%$ of the quiz correctly. If you have particular questions or a general problem in understanding this document, please contact Dr. W. Sturhahn, Bldg. 431, Rm. D007, tel. 0163.

\section{Principles of the Method}

In this chapter, we will discuss the basic properties of a nuclear resonance and its connection to atomic vibrations. In a study of the "Table of Isotopes," one can find several stable isotopes that have the first excited state at energies of a few keV. Such isotopes can be excited with x-rays, and observation of the scattering products reveals information about the nuclear resonance and its environment. In our experiment, we will work with the ${ }^{57} \mathrm{Fe}$ isotope, which has a nuclear resonance at $14.4125 \mathrm{keV}$. A copy of the nuclear level scheme of ${ }^{57} \mathrm{Fe}$ is attached. The natural abundance of ${ }^{57} \mathrm{Fe}$ is about $2 \%$, and we find numerous examples in technology, material science, life sciences, and environmental science, where iron compounds are of importance. The unique aspect of using resonant isotopes to measure phonon energies is mainly the selectivity. This means that vibrations can be probed locally in systems that have resonant isotopes in specific places, e.g., biomolecules like myoglobin, thin films, and materials under extremely high pressure (>100 GPa.)

An important property of the nuclear resonance is the energy width of the excited state

or natural line width. For ${ }^{57} \mathrm{Fe}$ we find the very small value of $\Gamma=4.66 \mathrm{neV}$. Only $\mathrm{x}$-ray sources with high spectral intensity have a reasonable chance of exciting the nucleus. A nucleus, once excited, decays with its natural lifetime of $\tau=\hbar / \Gamma=141 \mathrm{~ns}$. Even though the level width is small, the nuclear resonant cross section is large. We have $\sigma=2.56 \mathrm{Mb}$, which is 450 times larger than the cross section for photoelectric absorption by the atomic shell. 
The understanding of nuclear resonant scattering is much simplified by the ratios of the involved energy scales

transition energy $(\mathrm{keV}) \longleftrightarrow$ phonon energy $(\mathrm{meV}) \longleftrightarrow$ nuclear level width (neV) .

The energies are different by six orders of magnitude. Figure 1 illustrates this peculiar situation in more detail. In panel (a), the nucleus is held fixed, i.e., we neglect recoil from
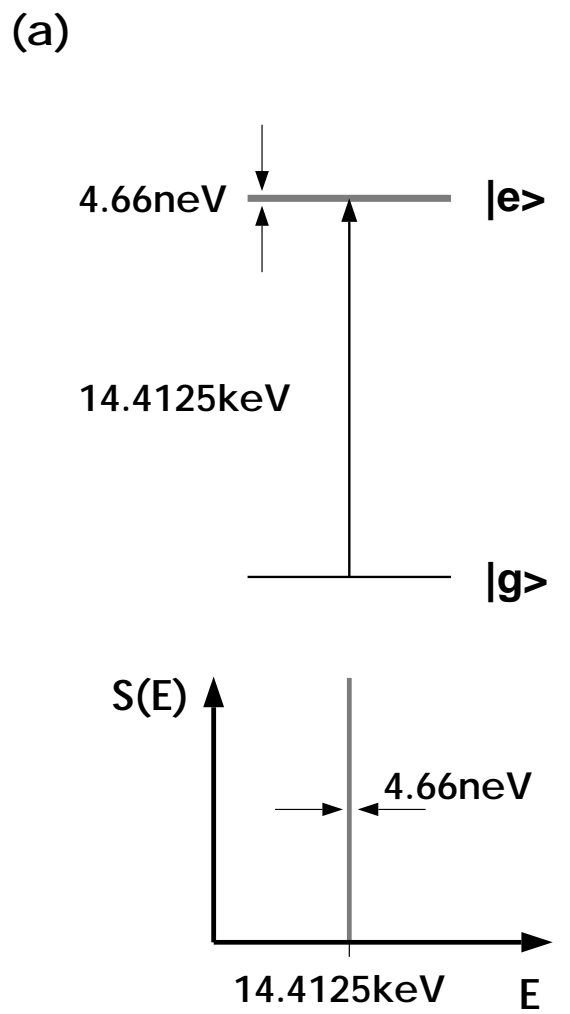

(b)
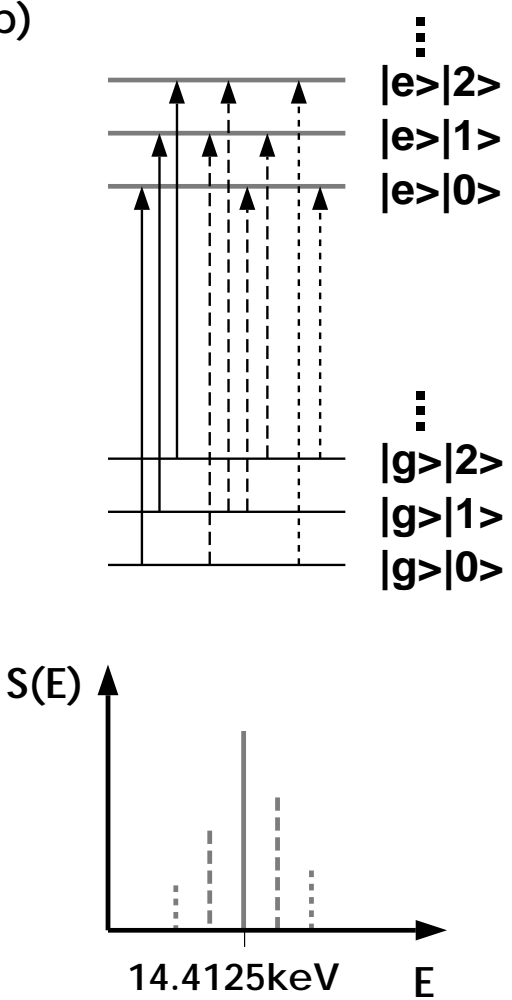

Figure 1: The influence of atomic vibration on the nuclear level scheme. Panel (a) shows the energy levels of a fixed ${ }^{57} \mathrm{Fe}$ nucleus. When x-rays interact with materials containing ${ }^{57}$ Fe the nuclear transition $|g\rangle \rightarrow|e\rangle$ causes a sharp resonance in the excitation probability density $S(E)$. The nucleus embedded in an Einstein solid has the more complex level scheme shown in panel (b). In addition to the peak at the nuclear transition energy, $S(E)$ now features side bands. The dashed lines show transitions that include creation or annihilation of one phonon with the Einstein energy. Transitions that involve more phonons are also possible, e.g., the dotted lines describe two-phonon creation/annihilation.

photon absorption, and only x-ray photons within width $\Gamma$ around the transition energy can excite the resonance. This behavior is quantitatively described by the excitation probability density $S(E)$, and the cross section for a particular scattering channel is proportional to $S(E)$. The value of $S(E) d E$ gives the probability that the nucleus can be 
excited by x-rays in the energy range $[E, E+d E]$. If the nucleus is allowed to oscillate with a particular frequency (the Einstein model of a solid), the picture changes dramatically. Panel (b) of Fig. 1 illustrates the new energy levels that evolve from combinations of nuclear states and phonon states, e.g., $|g\rangle|1\rangle$ is a quantum state with the nucleus in the ground state and one phonon present. The small coupling between nucleus, phonon, and $\mathrm{x}$-ray photon and the different energy scales mentioned before make such states very good approximations of the exact eigenstates of the coupled system. In a real material, phonons with many different energies exist, and the side bands in $S(E)$ become smeared out. However, the classification into one-phonon, two-phonon, etc., events remains valid. If the incident x-ray energy is too small to excite the nuclear resonance directly, phonons have to be annihilated. This process will always be less likely than the creation of phonons with the same energy. The ratio is given by the Boltzmann factor, i.e., $S(E)=\exp \left[E / k_{B} T\right] S(-E)$ with temperature $T$ and Boltzmann's constant $k_{B}$. At zero temperature the effect is

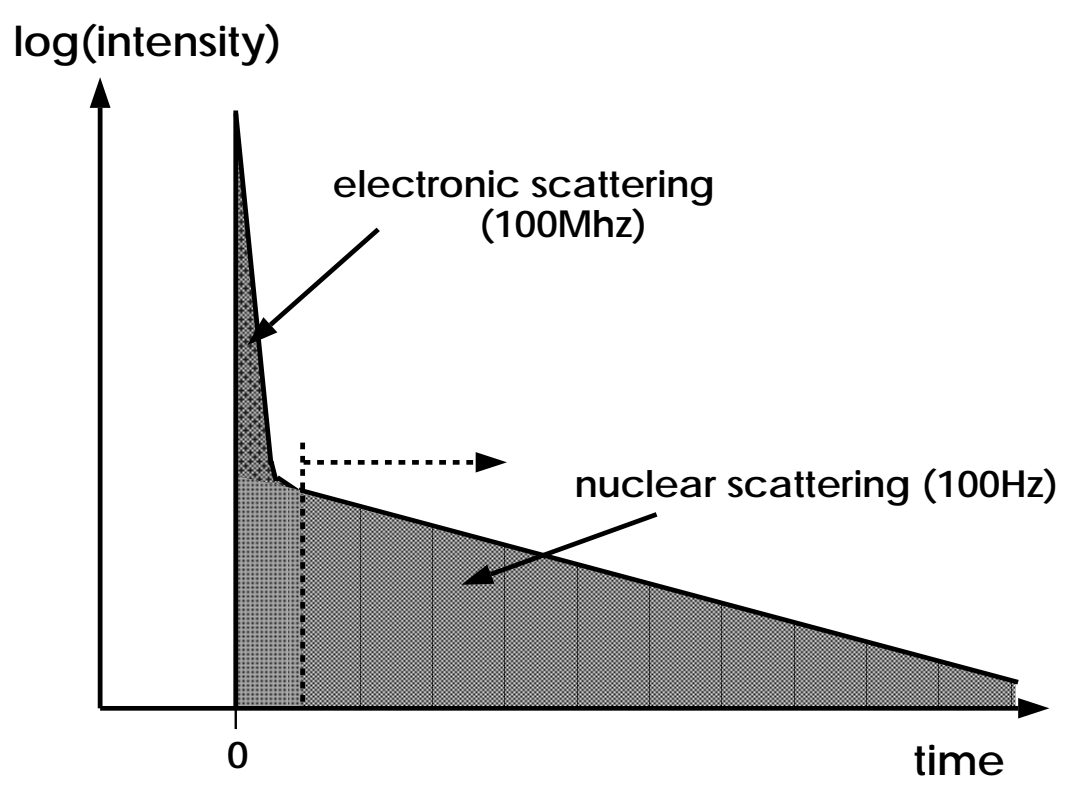

Figure 2: Scattered intensity vs. time after excitation. At time zero, a synchrotron radiation pulse excites a material containing a nuclear resonant isotope. The scattering from electrons is prompt, i.e., almost immediately after the pulse arrived. The response of the resonant nuclei is delayed. Time discrimination permits to distinguish nuclear and electronic scattering.

extreme because phonon annihilation becomes impossible, and $S(E)$ has only one sideband at positive energies.

If we can measure the probability of exciting a nucleus, we can derive $S(E)$, which contains all the phonon energies. Let us imagine a nucleus that was excited by some process. The nucleus will try to decay into the ground state either by emission of an x-ray photon of approximately $14.4125 \mathrm{keV}$ or by transfering the excitation energy to the electron shell. 
In the latter case, an electron is expelled (most likely from the K-shell), and the hole is quickly filled by other electrons under the emission of fluorescence x-rays. All these decay products are emitted with some delay relative to the time of excitation, and the average time is given by the natural life time of 141 ns. Figure 2 shows the scattered intensity of a material containing a nuclear resonance after excitation with a synchrotron radiation pulse. Scattering of $\mathrm{x}$-rays from electronic charge is very fast $\left(<10^{-12} \mathrm{~s}\right)$. If the energy of the incident x-rays is close to the nuclear transition energy, nuclei are excited, and delayed emission of x-rays can be observed. If only the delayed photons are counted while tuning the energy of the incident x-ray pulses, we expect to measure a function that is proportional to $S(E)$. The time discrimination removes all non-nuclear scattering of the x-rays very effectively. $S(E)$ is obtained from the measured data by proper normalization, and the partial phonon density of states can be extracted by a mathematical procedure. Both steps are explained in the attached paper Phonon Density of States Measured by Inelastic Nuclear Resonant Scattering. The word "partial" refers to the selection that has taken place by observing only vibrations at the positions of the resonant isotope.

\section{Setup and Components}

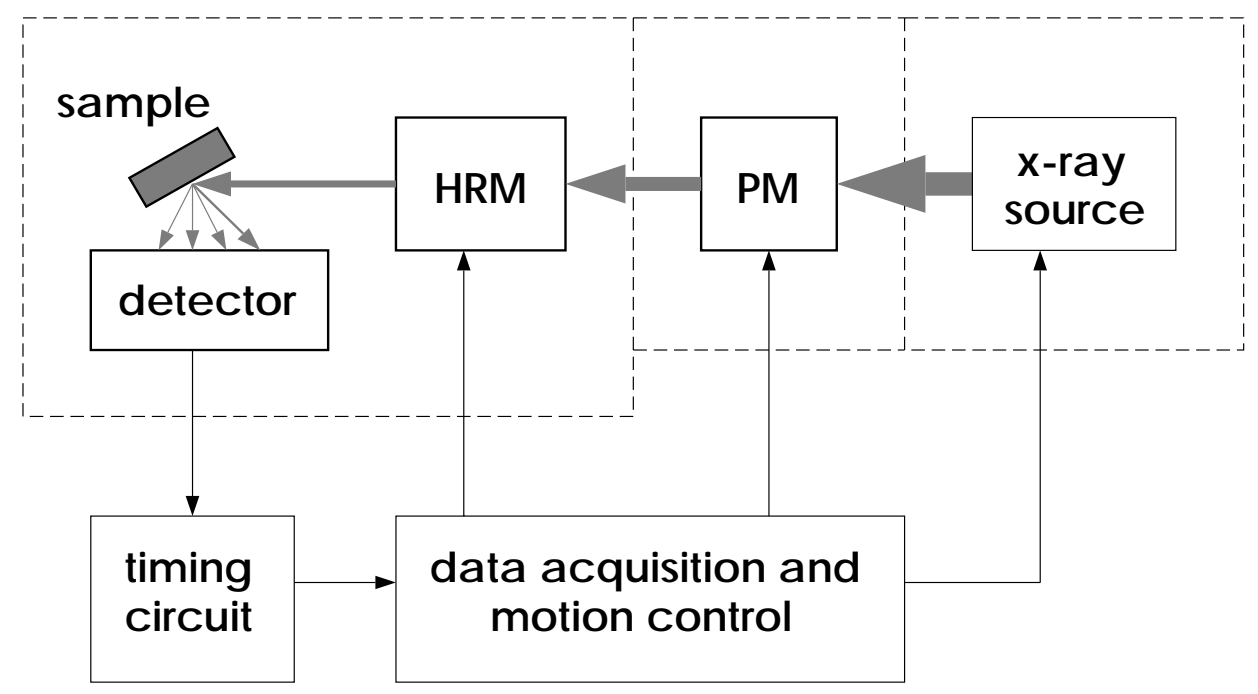

Figure 3: Setup of the experiment. Grey lines indicate x-rays. Solid lines symbolize flow of data or control information. PM and HRM abbreviate premonochromator and high-resolution monochromator, respectively. The dashed lines indicate enclosures with lead walls that protect experimenters from exposure to the x-rays. The components are described in the text.

The experimental setup can be broken down into several components. A schematic is shown in Fig. 3. At the beginning of the experiment all components will be aligned and 
functional. However, we require you to be familiar with the purpose and functionality of each component as described in this manual. The x-ray source are electrons that are orbiting in the storage ring and periodically (once per turn) pass through an undulator. The x-rays are monochromatized in two steps (premonochromator and high-resolution monochromator) to an energy bandwidth that is more narrow than the phonon spectrum of our samples. The monochromatized x-rays excite nuclear resonances in the sample. The re-emitted radiation is observed with the detector. The timing circuit measures the elapsed time between excitation and re-emission and removes prompt events. The control unit permits us to remotely interact with devices that are inside the radiation area and thus are not directly accessible during the measurement.

\section{X-ray source}

We use synchrotron radiation as the source for x-rays. Synchrotron radiation is created by charged particles that are stored on closed orbits. To keep the particles on closed orbits they have to be accelerated perpendicular to their propagation direction. At the Advanced Photon Source electrons with an energy of $7 \mathrm{GeV}$ are kept on an approximately circular orbit with a circumference of about $1.1 \mathrm{~km}(0.7$ miles $)$. The electrons are highly relativistic, and they need $3.68 \mu$ s for one turn. On average it takes about $10^{11}$ turns or 20 hours before an electron is lost by being scattered from its stable orbit. Therefore the storage ring is periodically refilled (usually twice a day.)

The magnets that keep the electrons in the orbit are called bending magnets. The x-rays produced at the bending magnets are moderately intense. Much more intense x-rays are obtained from undulators, which are devices with a spatially periodic magnetic field. The electrons propagate through the field and experience small periodic deviations from a straight line. The accelerations exerted by the periodic field lead to the emission of very collimated and intense x-rays. In the experiment, we use such an undulator to obtain the required spectral intensity (remember the narrow width of the nuclear resonance).

The electrons are not randomly distributed in the storage ring but may occupy a finite number of stable orbits. These stable orbits are called buckets, and there are 1296 buckets separated by $0.85 \mathrm{~m}$ or $2.84 \mathrm{~ns}$. Buckets that are filled with electrons are called bunches, and we call the actual pattern of bunches the time structure of the synchrotron radiation. The standard time structure at the Advanced Photon Source is shown in Fig. 4. It is very important for nuclear resonant scattering experiments that the time between bunches (150 ns) is larger than the dead time of the detector (20 ns.) Most bunches are filled with about $10^{11}$ electrons corresponding to an average current of $4 \mathrm{~mA}$.

The size of a bunch is $0.15 \mathrm{~mm}$ vertical, $0.5 \mathrm{~mm}$ horizontal, and $20 \mathrm{~mm}$ along the direction of propagation. The transverse size of the bunch determines the size of the x-ray source. Each bunch produces an x-ray pulse when passing through the undulator. The bunch length gives $70 \mathrm{ps}$ duration for each x-ray pulse. Another important parameter is the 


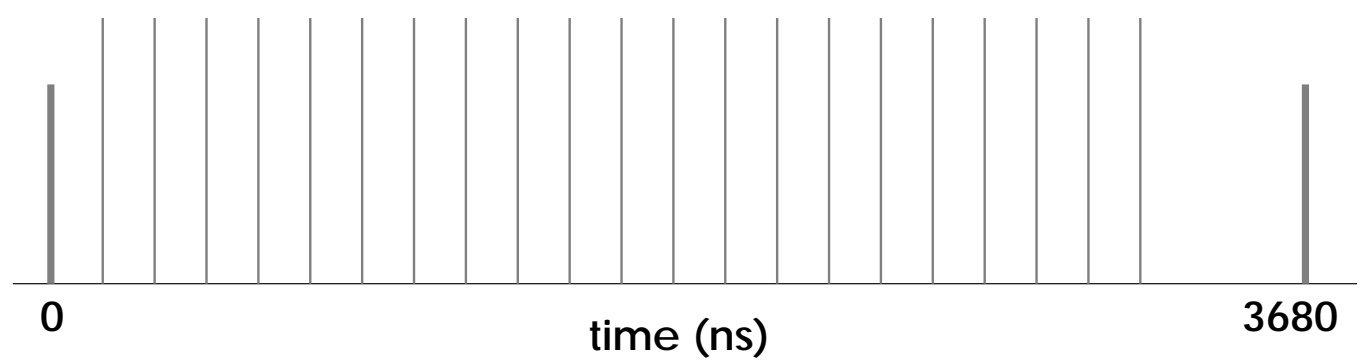

Figure 4: Standard time structure at the Advanced Photon Source. Grey vertical lines indicate the bunch positions. At time zero we find a group of six bunches separated by $2.84 \mathrm{~ns}$. The other bunches are singlets that are separated by $153 \mathrm{~ns}$. The gap on the right is $460 \mathrm{~ns}$ long. The bunch pattern is periodic and is repeated after $3680 \mathrm{~ns}$. The bunches in the sextet carry $3 \mathrm{~mA}$ current each. The singlet bunches contain $4 \mathrm{~mA}$ current.

divergence or angular width of the produced radiation. The divergence increases the size of the x-ray beam with larger distance from the undulator. We have divergences of $15 \mu \mathrm{rad}$ vertical and $40 \mu \mathrm{rad}$ horizontal. The sample is mounted at $35 \mathrm{~m}$ distance from the x-ray source (undulator). This distance results in a beam size of $0.7 \times 2 \mathrm{~mm}^{2}$.

\section{Premonochromator}

The undulator creates a broad energy band of x-rays (the so-called "white beam") that ranges from about $6 \mathrm{keV}$ up to several $100 \mathrm{keV}$, even though there are distinct peaks with enhanced intensity and widths of about $200 \mathrm{eV}$. The premonochromator filters an energy band of about $1 \mathrm{eV}$ from the white beam. This bandwidth is not narrow enough to perform phonon spectroscopy (remember that phonon energies are typically less than $0.1 \mathrm{eV}$ ) but the total power in the x-ray beam is reduced from $1000 \mathrm{~W}$ to $0.1 \mathrm{~W}$. The reduction in power is important because the high-resolution monochromator is very sensitive to temperature gradients (see next section). Two diamond crystals in nondispersive arrangement (like a "channel cut") operate at their (111) Bragg reflections to perform the task of filtering and power reduction. Diamond is a very suitable material because its excellent thermal conductivity and low absorption for x-rays. Each crystal's size is about $7 \times 5 \times 0.35 \mathrm{~mm}^{3}$. In the experiment, the premonochromator is adjusted to transmit around the nuclear resonance energy of $14.4125 \mathrm{keV}$. Also the undulator is tuned to produce an intense peak at this energy. The spectral photon flux after the premonochromator is $10^{13}$ photons $/ \mathrm{s} / \mathrm{eV}$ at $100 \mathrm{~mA}$ storage ring current.

\section{High-resolution monochromator}

In order to carry out your experiment, it is necessary to reduce the energy bandwidth to a level that will allow you to resolve vibrational excitations that range in energy from 0 
to say $100 \mathrm{meV}$. For this purpose, you will use a high-resolution mononchromator that reduces the energy bandwidth of the x-rays to approximately $2 \mathrm{meV}$ and has a tunability range that can easily cover the $200 \mathrm{meV}$ energy range where the probability for phonon excitation or annihilation is high.

The principle of high-resolution monochromatization in this region of the electromagnetic spectrum relies upon the use of Bragg diffraction from a series of perfect single crystalline materials. Pure single-crystalline silicon is the material most often used for this purpose because it is commercially available in large boules of extremely high quality. The high-resolution monochromator shown in Figs. 5 and 6 will be the one used in your experiment. The orientation of the four crystals is such that they will all be Bragg-diffracting the $14.4125 \mathrm{keV}$ x-rays from a pre-chosen set of diffraction planes. These diffraction planes and their orientation with respect to the crystal surfaces are chosen to achieve optimal performance in terms of efficiency and energy resolution and are selected when the monochromator is fabricated.
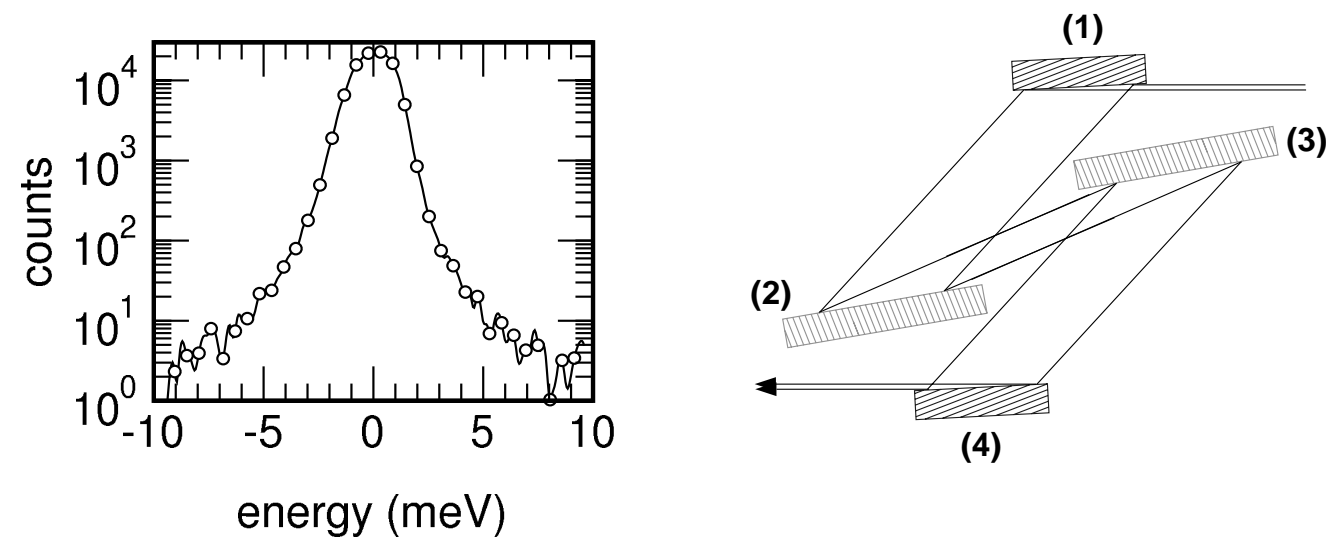

(4)

Figure 5: Design (right) and resolution function (left) of the high-resolution monochromator for $14.4125 \mathrm{keV}$ x-rays. The first and fourth crystal faces use the silicon (4 40 ) reflection, while the second and third faces use the silicon (9 75 ) reflection. Note that the diffraction planes are not parallel to the crystal surfaces. The resolution function was measured with coherent elastic nuclear resonant scattering relative to the nuclear transition energy.

The operation of the high-resolution monochromator will be relatively straightforward for this experiment since it will be pre-aligned for you. During the experiment, though, the high-resolution monochromator needs to be scanned in energy by changing the angles that the x-rays make with each crystal face. Also, the spacing of the diffraction planes depends upon the temperature, so any changes in the temperature of each crystal needs to be recorded. Crystal faces 1 and 4 are part of a single monolith. Faces 2 and 3 are part of another monolith. Crystals that are manufactured in this way are also called "channel cuts." Thus, there are only two rotation angles that need to be controlled. Calibration 


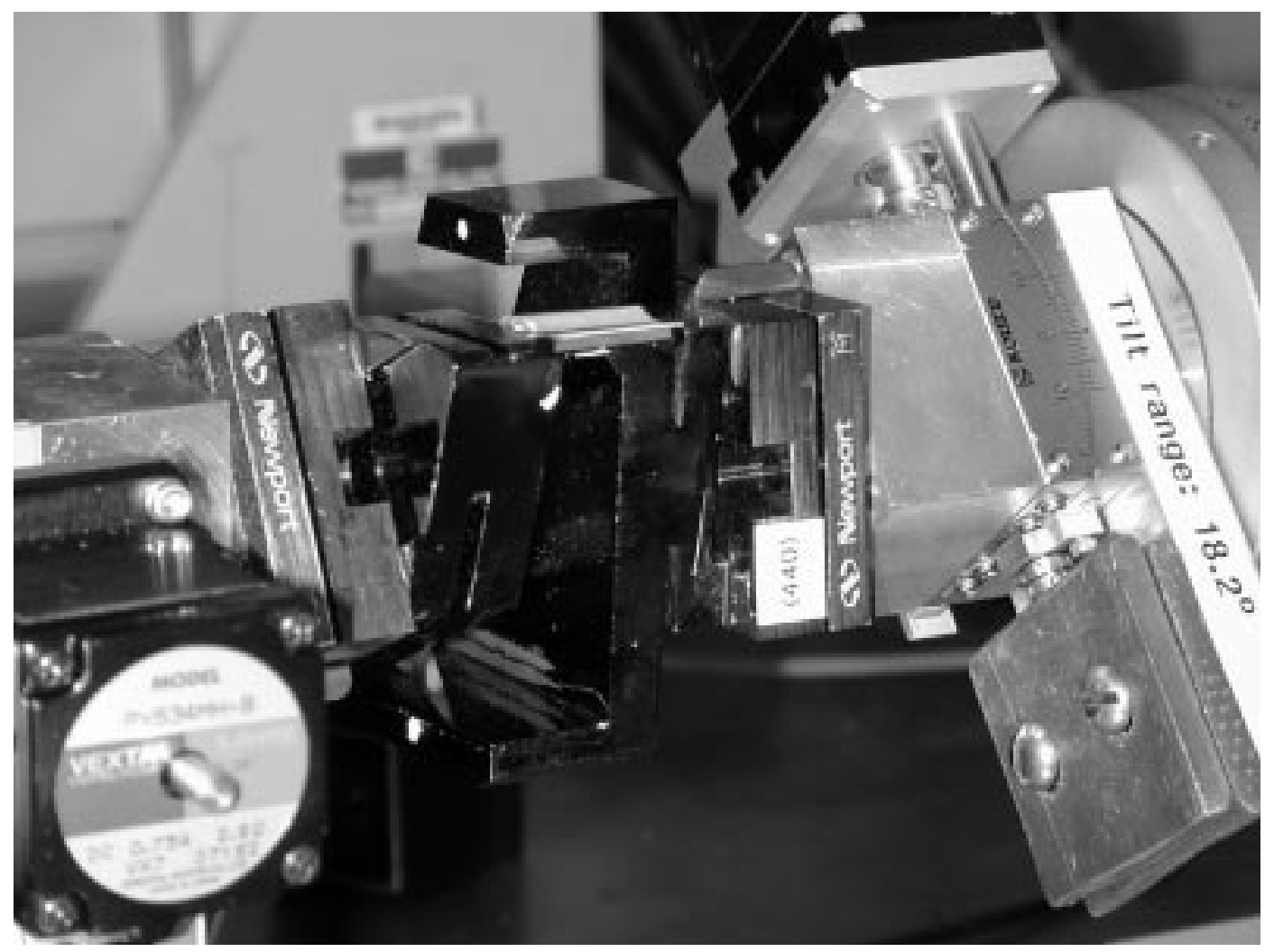

Figure 6: Picture of the high-resolution monochromator. The $\left(\begin{array}{lll}4 & 0\end{array}\right)$ channel cut is mounted on the right rotation stage. The incident x-rays are reflecting off the lower side (not visible) of the top silicon piece. The monochromatic beam leaves the device from the low lying surface of the same channel cut.

of the energy scale forms a critical aspect of the measurement, and the conversion from relative rotation angle and temperature of the two crystals to relative energy is given by

$$
\delta E=E_{o} \frac{\delta \theta_{1}-\delta \theta_{2}-\alpha\left(\delta T_{1} \tan \Theta_{1}+\delta T_{2} \tan \Theta_{2}\right)}{\tan \Theta_{1}+\tan \Theta_{2}},
$$

where the energy, angles and temperatures are referenced from their values at the nuclear transition energy. The nominal Bragg angle of each crystal is given by $\Theta_{1}=26.6^{\circ}$ and $\Theta_{2}=80.4^{\circ}$, the temperature of the $i^{\text {th }}$ crystal is depicted by $T_{i}$, the coefficient of thermal expansion $\alpha$ has the value $2.56 \times 10^{-6} K^{-1}$ for silicon near room temperature, and $E_{o}=14.412497 \mathrm{keV}$.

In order to take high-quality spectroscopic data, it is necessary to know the energy response of your instrument. In the case of inelastic nuclear resonant scattering with synchrotron radiation, energy response is determined solely by the high-resolution monochromator. The energy resolution function of the monochromator can be measured using 
coherent elastic nuclear resonant scattering and is used in the evaluation process of your inelastic data. You will be provided with the resolution function of the monochromator. An example of an energy resolution function for a high-resolution monochromator is given in figure 5 .

\section{Detector and timing circuit}

We discussed earlier how the nuclear resonant signal is discriminated by timing technique (see Fig. 2). We need a detector with a very good time resolution, say $1 \mathrm{~ns}$. Also an excellent dynamic range is required, i.e., after the intense pulse of prompt photons the detector has to quiet down in a reasonably short time, say $10 \mathrm{~ns}$. The best x-ray detectors
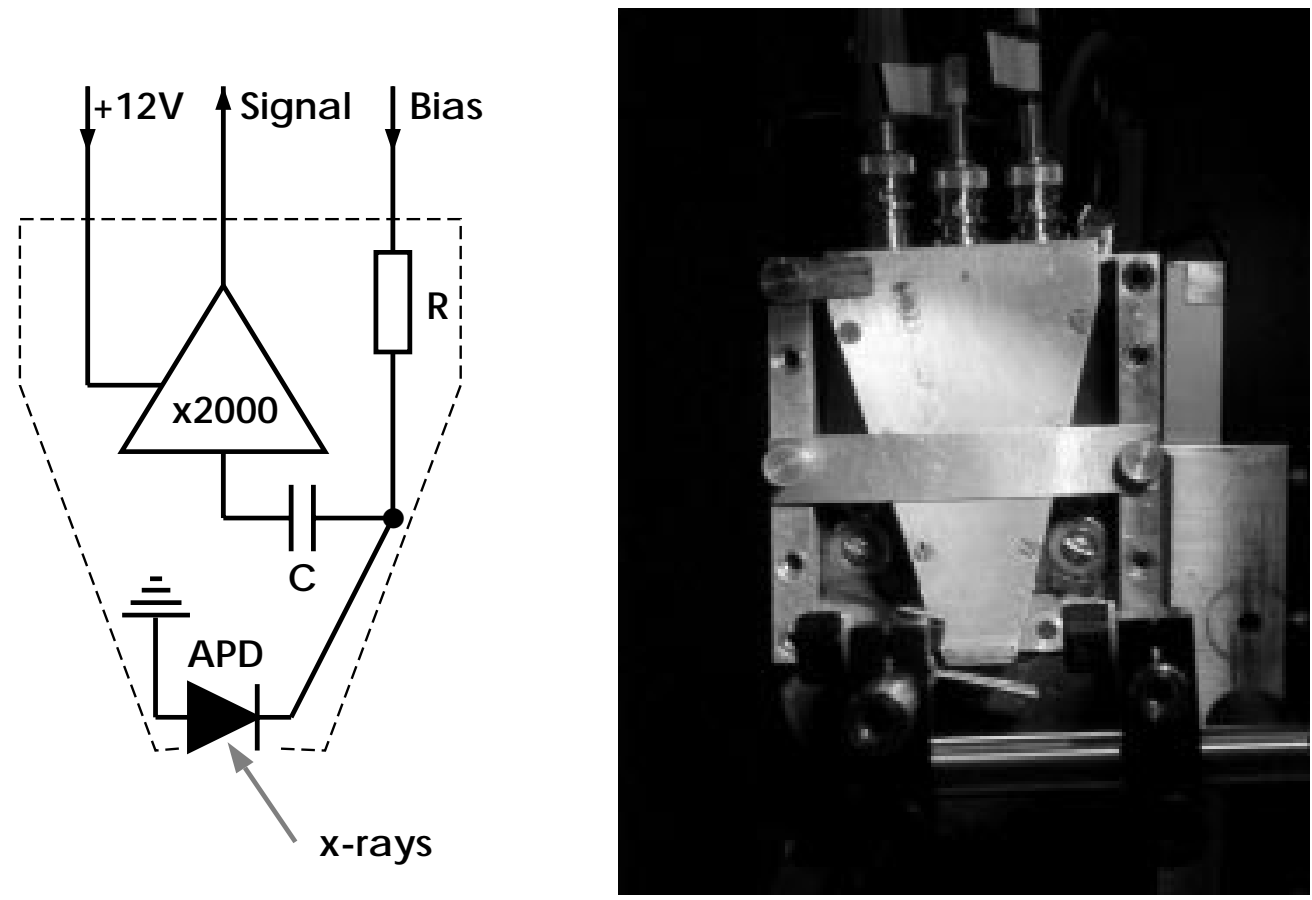

Figure 7: Circuit diagram of the APD-detector (left) and picture of the actual device (right). The bias voltage varies with the particular APD around typically $300 \mathrm{~V}$. Other typical values are $\mathrm{R}=10 \mathrm{M} \Omega$ and $\mathrm{C}=100 \mathrm{pF}$. The signal following the absorption of a $14.4 \mathrm{keV}$ photon is a negative pulse of $3 \mathrm{~ns}$ duration and $200 \mathrm{mV}$ height on $50 \Omega$.

with such qualities are avalanche photodiodes or APDs. The APD is a semiconductor device made from silicon. It consists of a p-n junction that is operated under reverse bias. The electric fields in a region around this $\mathrm{p}-\mathrm{n}$ junction are high enough to amplify a small current from impact ionization, e.g., absorption of an x-ray photon. This so called "active region" is about $100 \mu \mathrm{m}$ thick. The active area of the APD is $10 \times 10 \mathrm{~mm}^{2}$. Figure 7 shows a circuit diagram and a picture of the actual detector. The detector produces negative signals of a few ns duration. The height of the pulse depends on the x-ray energy. For a 
$14.4 \mathrm{keV}$ photon, the pulse height is about $200 \mathrm{mV}$ on $50 \Omega$. The time resolution of this detector is about $1 \mathrm{~ns}$.

Additional electronic components are needed to discriminate between prompt and delayed $\mathrm{x}$-rays. First we need to know when the x-ray pulses arrive at the sample. We learned in the section about the $\mathrm{x}$-ray source that $\mathrm{x}$-ray pulses will be synchronized with the bunch pattern in the storage ring. The bunch pattern can be derived electronically from the operation parameters of the storage ring. The device that performs this task is called the bunch clock generator. It provides us with a sequence of electronic pulses that looks just like Fig. 4. The timing circuit will compare the output signal of the APD detector with the signal from the bunch clock generator. Panel (a) in Fig. 8 shows the circuit. The

(a)

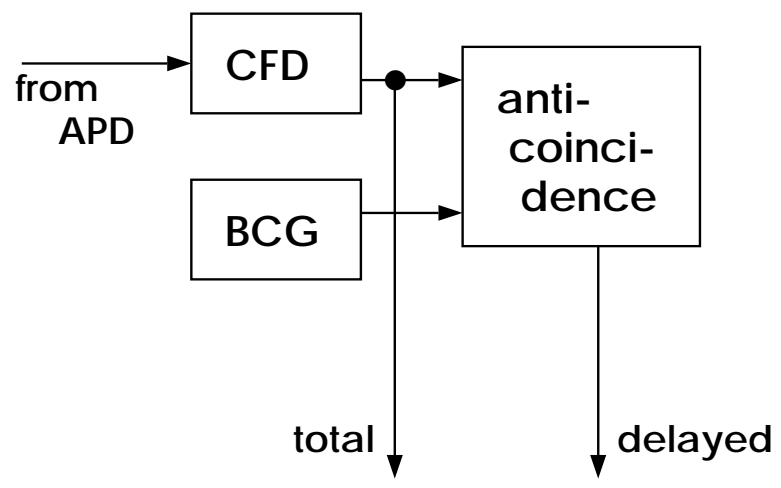

(b)

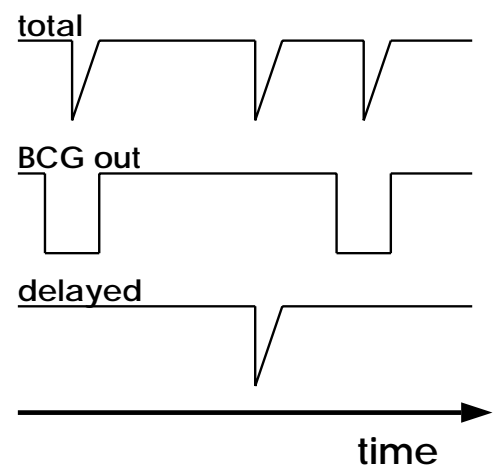

Figure 8: Timing circuit for the discrimination of prompt and delayed photons. The analog signal from the APD-detector is converted to a digital NIM-signal by the constantfraction discriminator (CFD.) An anti-coincidence logic blanks out the prompt signals that arrive synchronously with the bunch clock generator (BCG) output. Only delayed pulses remain as output signal. The timing diagram is shown in panel (b).

anti-coincidence unit filters out the delayed events. The timing diagram is displayed in panel (b). Different experimental situations are accommodated by the adjustable width and delay time of the output pulses of the bunch clock generator. Besides delayed events that originate in nuclear resonant scattering, electronic noise may fake "delayed events" because such pulses are asynchronous with the bunch pattern. Electronic noise created by the detector is unavoidable but, for our APD detectors, sufficiently small. Usually $0.03 \mathrm{cps}$ are caused by noise. The noise events will be independent of the energy of the incident $\mathrm{x}$-rays (remember that the nuclear delayed event will strongly depend on the incident energy) and can be measured by tuning the incident x-ray energy sufficiently far off resonance. 


\section{Experimental Procedure}

First and foremost, think before you act. Familiarize yourself with the beamline setup. The experiment to be performed will involve measuring the nuclear resonant absorption probability of different materials around the $14.4 \mathrm{keV}$ nuclear resonance using the technique of inelastic nuclear resonant scattering. You will perform this measurement on three different ${ }^{57} \mathrm{Fe}$-containing compounds, iron metal, stainless steel $\left(\mathrm{Fe}_{55} \mathrm{Cr}_{25} \mathrm{Ni}_{20}\right)$, and hematite $\left(\mathrm{Fe}_{2} \mathrm{O}_{3}\right.$.) Naturally occurring iron only contains $2 \%$ of ${ }^{57} \mathrm{Fe}$, the rest being predominantly ${ }^{56} \mathrm{Fe}$. The samples you will measure are enriched to greater than $95 \%{ }^{57} \mathrm{Fe}$ to increase your signal so that you may complete the measurements in the alloted time.

Performing the measurement will involve measuring the yield of nuclear resonant photons as a function of incident x-ray energy. To measure the photons you will use an avalanche photodiode detector that is placed as close as possible to the scattering volume. Due to the $141 \mathrm{~ns}$ lifetime of the $14.4 \mathrm{keV}$ nuclear excited state, the nuclear resonant photons are "delayed" relative to the nonresonant, or "prompt", photons. Timing electronics are used to separate the nuclear resonant delayed photons from the nonresonant prompt photons. The timing electronics will be set up beforehand, but you should understand the importance of the time-filtering technique, as well as the essential properties of the detector that motivates one to choose it over other detectors. The energy of the x-rays incident on the sample is varied by rotating the crystals of the high-resolution monochromator. To change the energy transmitted by the monochromator by $1 \mathrm{meV}$, you need to rotate the $(440)$ crystal by $0.035 \mu \mathrm{rad}$ and the $(975)$ crystal by $0.48 \mu \mathrm{rad}$. Note that temperature changes of the high-resolution monochromator will also alter the transmitted x-ray energy in accordance with equation 1, so thermal stability of the monochromator is also important. You should record the crystal angles as measured by the number of pulses transmitted to the stepper motors controlling the rotation axes, as well as the temperatures of the crystals as measured by thermal sensors at every step of the energy scan. This will allow you to reconstruct the energy scale later by using Eq. (1).

Nearly everything in the experimental hutch is remotely controlled by computer software. The crystal angles can be scanned with the use of the computer. In addition, you should setup the computer to have all the necessary experimental parameters (angles, temperatures, countrates, etc.) written to a data file. In addition, there are many sensors/detectors/monitors that allow one to track the state of the x-ray beam, the optics, and the experiment itself. Some of these need to be checked before the experiment can be performed. The following is a small checklist that you should go through to ensure that you collect usable data.

\section{Checklist for collecting data}

1. Beam should be stable

$\longrightarrow \leq 5 \mu \mathrm{rad}$ vertical angle 
$\longrightarrow \leq 25 \mu \mathrm{rad}$ horizontal angle

$\longrightarrow \leq 100 \mu \mathrm{m}$ vertical position

$\longrightarrow \leq 100 \mu \mathrm{m}$ horizontal position

2. Premonochromator should be aligned properly

$\longrightarrow$ counting rate (flux) after premono should be optimized with a drift of $\leq 5 \% / \mathrm{hr}$

3. High-resolution monochromator (HRM) should be aligned properly

$\longrightarrow$ maximize flux after HRM by rotating the crystals while maintaining the energy alignment

$\longrightarrow$ move energy of HRM to nuclear resonance energy and optimize delayed counting rate

$\longrightarrow$ counting rate (flux) after HRM should have a drift of $\leq 5 \% / \mathrm{hr}$

4. Timing electronics should be set properly

$\longrightarrow$ move energy of HRM $200 \mathrm{meV}$ below the nuclear resonance energy and measure background (delayed) counting rate in the APD detector: should be $\leq 0.03 \mathrm{cps}$

$\longrightarrow$ move energy of HRM back to nuclear resonance energy

5. Scan parameters should be set properly

$\longrightarrow$ scan range should be properly centered and extending at least $\pm 100 \mathrm{meV}$

$\longrightarrow$ energy step size should be approximately one third of the energy bandwidth of the HRM

$\longrightarrow$ counting time per energy step should be adjusted to produce an hour-long scan

$\longrightarrow$ check that all relevant experimental parameters necessary to evaluate the data are being written to the data file: motor positions, crystal temperatures, delayed counts, incident intensity on sample etc.

\section{Additional Reading}

- The following material has to be read.

1. Lecture notes by S. K. Sinha, Inelastic X-ray Scattering

2. W. Sturhahn et al., Phonon Density of States Measured by Inelastic Nuclear Resonant Scattering, Phys.Rev.Lett. 74, 3832 (1995)

- These texts provide a good source of supplementary information.

1. Phonons in general :

N.W. Ashcroft and N.D. Mermin, Solid State Physics, (W.B. Saunders Company, Philadelphia, PA, 1976) 
2. Inelastic x-ray scattering in general :

E. Burkel, Phonon spectroscopy by inelastic x-ray scattering, Rep.Prog.Phys. 63, $171(2000)$

3. Nuclear absorption :

K.S. Singwi and A.Sjölander, Resonance Absorption of Nuclear Gamma Rays and the Dynamics of Atomic Motions, Phys.Rev.120, 1093 (1960)

4. Procedure of data evaluation :

M.Y.Hu et al., Data Analysis for Inelastic Nuclear Resonant Absorption Experiments with Synchrotron Radiation, Nucl.Instrum.Methods A 428 (1999) 551

5. Software for data evaluation :

W. Sturhahn, CONUSS and PHOENIX: Evaluation of Nuclear Resonant Scattering Data Hyp.Int. 125, 149 (2000), (the second part of the paper)

- More details can be found in the following papers.

1. Monochromators :

T.S. Toellner, Monochromatization of synchrotron radiation for nuclear resonant scattering experiments, Hyp.Int. 125, 3 (2000)

2. Theory :

W.Sturhahn and V.Kohn, Theoretical Aspects of Inelastic Nuclear Resonant Scattering, Hyp.Int. 123/124, 367 (1999)

3. Experiment :

A.I.Chumakov and W.Sturhahn, Experimental Aspects of Inelastic Nuclear Resonant Scattering, Hyp.Int. 123/124, 781 (1999)

\section{Quiz}

The following short quiz tests how carefully you have read the given material and sometimes a little more. You should be able to answer $75 \%$ or so correctly. We will discuss the answers before the experiments start.

1. What is the abundance of the element iron in earth's crust?

2. Why is the planned experiment selective to ${ }^{57} \mathrm{Fe}$ ?

3. What are energy and lifetime of the first nuclear excited state of ${ }^{57} \mathrm{Fe}$ ? What are energy and lifetime of an excited Fe atom with a hole in the K-shell?

4. Why is the nuclear resonant cross section so large? 
5. Imagine the resonant isotope embedded in an isotropic Debye solid (see Fig. 1 for the case of the Einstein solid.) What are the width and the height of the peak in $S(E)$ that occurs at the exact nuclear transition energy?

6. We normalize our data using the first moment instead of the zeroth moment (area). Why can't we simply use area normalization to obtain $S(E)$ from our data?

7. Many solids behave like a collection of coupled harmonic oscillators, and $S(E)$ can be expressed as sum of $n$-phonon contributions $(n=0,1, \ldots)$ Calculate the onephonon contribution $S_{1}(E)$ for the Debye model. Give the functional dependence of $S_{1}(E)$ for $E \ll k_{B} T$.

8. Why can't we use an x-ray tube for our experiment?

9. What is the task of the premonochromator?

10. Compare the power density $\left(\mathrm{W} / \mathrm{mm}^{2}\right)$ of white beam at $25 \mathrm{~m}$ from the undulator and sunlight in Chicago in summer.

11. Why is the overall energy resolution only determined by the high-resolution monochromator?

12. Equation (1) implies that we can change $\delta \theta_{1}$ and $\delta \theta_{2}$ without changing the energy. What does this mean?

13. Why do we need the resolution function of the monochromator?

14. What are the properties that make APDs suitable detectors for our experiment? Do we need energy resolution in the detector?

15. Calculate the temperature change (assume the same value for both crystals) that is needed to shift the energy of the high-resolution monochromator by $1 \mathrm{meV}$.

16. Why is it better to move the HRM $200 \mathrm{meV}$ below (rather than above) the nuclear resonance energy to measure the background? 